\title{
UK Renal Registry 20th Annual Report: Chapter 11 Clinical, Haematological and Biochemical Parameters in Patients on Renal Replacement Therapy in Paediatric Centres in the UK in 2016: National and Centre-specific Analyses
}

\author{
Lucy Plumb ${ }^{\mathrm{ab}}$, Anna Casula ${ }^{\mathrm{a}}$, Winnie Magadi ${ }^{\mathrm{a}}$, Fiona Braddon ${ }^{\mathrm{a}}$, Malcolm Lewis ${ }^{c}$, \\ Stephen D Marks ${ }^{d}$, Mohan Shenoy ${ }^{e}$, Manish D Sinha ${ }^{f}$, Heather Maxwell ${ }^{9}$. \\ ${ }^{a}$ UK Renal Registry, Bristol, UK; ${ }^{b}$ University of Bristol, Bristol, UK; ${ }^{c}$ Children's University Hospital, Temple Street, Dublin, \\ Ireland; ${ }^{d}$ Great Ormond Street Hospital for Children NHS Foundation Trust, London, UK; ${ }^{e}$ Royal Manchester Children's \\ Hospital, Manchester, UK; 'Evelina London Children's Hospital, London, UK; ${ }^{9}$ Royal Hospital for Children, Glasgow, UK
}

\section{Keywords}

Adolescents - Biochemical variables - Blood pressure - Body mass index - Children - Dialysis - Established renal failure . Growth · Haemoglobin · Height - Hypertension · Paediatric . Quality improvement . Renal replacement therapy . Transplant $\cdot$ Weight $\cdot$ Young adults

\section{Summary}

- In 2016, the median height $\mathrm{z}$-score for prevalent paediatric patients on dialysis was -1.8 and -1.1 for those with a functioning transplant $(p<$ $0.0001)$.

- The median weight $\mathrm{z}$-score for children receiving dialysis in 2016 was -1.2 compared with -0.2 for children with a functioning transplant.

- The median systolic blood pressure (SBP) z-score for transplanted children was 0.3 compared with 0.9 for dialysis patients $(p<0.0001)$.
- Of those with complete data, $72 \%$ of the prevalent paediatric renal replacement therapy (RRT) population in 2016 had one or more risk factors for cardiovascular disease; $6 \%$ had three risk factors present.

- All centres reported quarterly laboratory data in 2016. Quarterly data for transplant patients revealed a median creatinine of $77 \mu \mathrm{mol} / \mathrm{L}$. Data for dialysis patients revealed median values within the normal range for most biochemical and laboratory parameters, however wide inter-centre variation was seen for parameters such as ferritin and phosphate. Evidence of hyperparathyroidism (median parathyroid hormone (PTH) $16.5 \mathrm{pmol} / \mathrm{L}$ ) was noted within this cohort.

- Most (91\%) prevalent transplant patients achieved the national haemoglobin standard in 2016: 86\% had a normal range bicarbonate level and $81 \%$ had a PTH within acceptable range.

- For prevalent patients on haemodialysis in 2016, $56 \%$ achieved SBP values of less than the 90th percentile. Achievement of standards was $71 \%$,

Lucy Plumb

UK Renal Registry, Southmead Hospital, Southmead Road,

Bristol, BS10 5NB, UK

Email: renalregistry@renalregistry.nhs.uk
Fax +41613061234 E-Mail karger@karger.com www.karger.com/nef 
$79 \%, 54 \%$ and $41 \%$ for haemoglobin, calcium, phosphate and PTH respectively.

- For prevalent patients on peritoneal dialysis in 2016, $65 \%$ achieved SBP values of less than the 90th percentile. Achievement of standards was 77\%, 69\%, $59 \%$ and $35 \%$ for haemoglobin, calcium, phosphate and PTH standards respectively.

\section{Introduction}

This chapter focuses on the following variables for the prevalent paediatric dialysis and transplantation cohort on 31 December 2016:

1. The completeness of data returns to the UK Renal Registry (UKRR)

2. Anthropometric characteristics and growth

3. Cardiovascular risk factors (CVRFs)

4. Laboratory, clinical and biochemical indices relevant to the management of established renal failure.

Analyses are reported for prevalent patients aged $<18$ years managed in paediatric centres receiving chronic RRT for the year 2016 and for the time period 2002-2016 (inclusive).

\section{Methods}

Processes for data collection for the paediatric UKRR are described in chapter 4 . The data presented in this chapter relate to the annual census date of 31 December 2016.

Standards and standardisation

Standards are in bold text and are from the Renal Association's (2002) Treatment of adults and children with renal failure: standards and audit measures (third edition) [1], unless otherwise stated.

Data for height, weight, body mass index (BMI) and SBP vary with age, sex and size and are therefore presented as z-scores.

\section{Anthropometry}

'Measures of supine length or standing height and weight should be monitored at each clinic visit. All measurements should be plotted on European reference growth charts for healthy children.'

The reference range for height, weight and body mass index in childhood varies with gender and age. BMI was calculated using the formula BMI = weight $(\mathrm{kg}) /$ height $^{2}(\mathrm{~m})$. Height and weight were adjusted for age. To account for discrepancies in linear growth secondary to renal disease, BMI was expressed according to height-age, rather than chronological age. The International Obesity Taskforce definition [2] was used to define overweight and obesity; z-scores were calculated based on the British 1990 reference data for height and weight [3].

Blood pressure

'Blood pressure varies throughout childhood and should be maintained within two standard deviations of the mean for normal children of the same height and sex. The systolic blood pressure during peritoneal dialysis or after haemodialysis should be maintained at $<90$ th centile for age, gender and height.'

'In paediatric renal transplant patients, the systolic blood pressure should be maintained at $<90$ th percentile for age, gender and height.'

The analyses of systolic blood pressure in this report describe the achievement of values at or below the 90th percentile. Guidance for blood pressure in paediatric renal transplant patients was based on 2011 British Association for Paediatric Nephrology recommendations [4].

The reference range for SBP varies with gender, age and height. The data are therefore presented as z-scores based on data from the fourth report of the National High Blood Pressure Education Programme working group in the United States [5].

\section{Cholesterol}

The National Heart Lung and Blood Institute recommends screening children at risk of secondary dyslipidaemias including those with chronic kidney disease (CKD) [6]. Given the potential long-term cardiovascular benefit and minimal harm associated with testing, this recommendation was endorsed by the Kidney Disease Improving Global Outcomes (KDIGO) working group. Their 2013 clinical guidance on lipid management recommends annual measurement of fasting lipid levels in children with CKD, including those on RRT [7]. Both organisations consider a high total cholesterol as $\geqslant 5.2 \mathrm{mmol} / \mathrm{L} \mathrm{[6]}$. This cut-off has been adopted for this report.

\section{Haemoglobin and Ferritin}

The National Institute for Health and Care Excellence (NICE) guidance on the management of anaemia in adults and children with CKD was updated in 2015 and was used to describe haemoglobin ( $\mathrm{Hb})$ findings for this report [8].

'Typically maintain the aspirational $\mathrm{Hb}$ range between 100 and $120 \mathrm{~g} / \mathrm{L}$ for young people and children aged 2 years and older, and between 95 and $115 \mathrm{~g} / \mathrm{L}$ for children younger than 2 years of age, reflecting the lower normal range in that age group.'

New recommendations suggest that ferritin alone is insufficient for assessment of iron deficiency status. Isolated serum ferritin however may be used to guide maximum iron levels.

'Do not request transferrin saturation or serum ferritin measurement alone to assess iron deficiency status in people with anaemia of CKD.'

'In people treated with iron, serum ferritin levels should not rise above 800 micrograms/litre. In order to prevent this, review the dose of iron when serum ferritin levels reach 500 micrograms/litre.' 
Table 11.1. Summary of relevant biochemical clinical audit measures

\begin{tabular}{lccc}
\hline & \multicolumn{3}{c}{ Age (years) } \\
\cline { 2 - 4 } Parameter & $<1$ & $1-5$ & $6-12$ \\
\hline $\mathrm{Hb}(\mathrm{g} / \mathrm{L})$ & $\begin{array}{c}\text { Maintain } 95-115 \text { if } \\
\text { aged }<2 \text { years }\end{array}$ & $\begin{array}{c}\text { Maintain } 100-120 \text { if } \\
\text { aged }>2 \text { years }\end{array}$ & $100-120$ \\
Ferritin $(\mu \mathrm{g} / \mathrm{L})$ & $200-500$ & $200-500$ & $200-500$ \\
Corrected calcium $(\mathrm{mmol} / \mathrm{L})$ & $2.24-2.74$ & $2.19-2.69$ & $2.19-2.69$ \\
Phosphate $(\mathrm{mmol} / \mathrm{L})$ & $1.10-1.95$ & $1.05-1.75$ & $1.05-1.75$ \\
PTH (individual centre) & & Within twice the normal range & $2.15-2.55$ \\
Bicarbonate $(\mathrm{mmol} / \mathrm{L})$ & Levels may be maintained within normal range if growing appropriately \\
\hline
\end{tabular}

$\mathrm{Hb}$ - haemoglobin; PTH - parathyroid hormone

In view of this, the reporting of ferritin in relation to the recognition of anaemia has been removed from this year's report. Quarterly ferritin data by centre is reported; this should only be interpreted in the context of maximum iron levels. $\mathrm{Hb}$ and ferritin were analysed using age-related laboratory reference ranges as in table 11.1.

\section{Calcium, phosphate and parathyroid hormone}

'Serum phosphate and calcium should be kept within the normal range. PTH levels should be maintained within twice the upper limit of the normal range but, contrary to adult standards, may be kept within the normal range if growth is normal.'

Calcium, phosphate and PTH were analysed using agerelated laboratory reference ranges as in table 11.1. Individual variable data analysis has been performed by centre and nationally. It should be noted that 'normal' growth is difficult to determine in relation to paediatric established renal failure (ERF).

\section{Bicarbonate}

'Serum bicarbonate concentrations should be between 20 and $26 \mathrm{mmol} / \mathrm{L}$.'

Bicarbonate reference ranges varied by centre and are reported as within or outside the reference range as given in table 11.1.

\section{Cardiovascular risk factors (CVRFs)}

A cross-sectional assessment of the prevalence of risk factors for cardiovascular disease in paediatric patients with ERF was performed. Risk factors described include hypertension, overweight/obesity and hypercholesterolaemia. Evidence pertaining to childhood CVRFs and their association with long-term cardiovascular risk is available from The National Heart Lung and Blood Institute [6].

\section{Statistical analyses}

Annual and quarterly clinical and laboratory data have been analysed separately, with annual data being used unless stated otherwise. Data were analysed to calculate summary statistics (maximum, minimum, mean and median values in addition to standard deviation and interquartile ranges). Where applicable, the percentage achieving the audit standard was also calculated. If a patient had missing data, they were excluded from the relevant analyses. Centre-specific data for each UK paediatric nephrology centre is presented where completeness reaches at least $50 \%$.

Estimated glomerular filtration rate (eGFR) was calculated using the updated 'bedside' Schwartz formula [9], using centrespecific individual correction factors submitted to the UKRR. Caution should be taken when interpreting results based on a single annual measurement per patient. Furthermore, additional factors that may impact the analysis include the assays used by centres and timing of blood results in relation to RRT. All analyses were performed using SAS 9.3.

\section{Results}

\section{Data completeness}

\section{Annual data}

Tables 11.2 and 11.3 show the completeness of annual data returns for transplant and dialysis prevalent patients in 2016.

Data returns for 2016 were lower than previous years for key variables such as height, weight and systolic blood pressure in both transplant and dialysis patients. As in previous years, transplant patients tended to have better data completeness for these variables, although this too has fallen. Haematological and biochemical parameters collected (including haemoglobin, creatinine, bicarbonate and PTH) had $>90 \%$ completeness overall for both groups of patients. In 2016, completeness of erythropoietin stimulating agent (ESA) and IV iron use was extremely poor, with many sites not submitting any data. Analyses including these variables have therefore been removed from this year's report. Growth hormone use (data not shown) had $18 \%$ completeness and was also omitted from analyses. 
Table 11.2. Percentage data completeness for transplant patients $<18$ years old by centre for each variable and total number of patients per centre on 31/12/2016, (annual data return)

\begin{tabular}{|c|c|c|c|c|c|c|c|c|c|c|c|c|c|c|c|}
\hline \multirow[b]{2}{*}{ Centre } & \multicolumn{15}{|c|}{ Transplant patients } \\
\hline & $N$ & Height & Weight & BMI & SBP & $\mathrm{Hb}$ & Creat & Ferr & ESA & IV iron & Chol & Bicarb & PTH & $\mathrm{Ca}$ & Phos \\
\hline Bham_P & 89 & 82.0 & 82.0 & 82.0 & 84.3 & 93.3 & 97.8 & 49.4 & 0.0 & 0.0 & 65.2 & 96.6 & 93.3 & 97.8 & 97.8 \\
\hline Blfst_P & 22 & 95.5 & 100.0 & 95.5 & 95.5 & 90.9 & 90.9 & 81.8 & 90.9 & 90.9 & 86.4 & 90.9 & 86.4 & 90.9 & 90.9 \\
\hline Brstl_P & 40 & 95.0 & 97.5 & 92.5 & 97.5 & 100.0 & 100.0 & 67.5 & 100.0 & 100.0 & 60.0 & 97.5 & 77.5 & 100.0 & 100.0 \\
\hline Cardf_P & 26 & 88.5 & 92.3 & 84.6 & 92.3 & 96.2 & 96.2 & 88.5 & 7.7 & 7.7 & 84.6 & 96.2 & 88.5 & 96.2 & 96.2 \\
\hline Glasg_P & 43 & 100.0 & 100.0 & 100.0 & 100.0 & 86.1 & 86.1 & 62.8 & 100.0 & 100.0 & 44.2 & 86.1 & 83.7 & 86.1 & 86.1 \\
\hline L Eve_P & 80 & 98.8 & 98.8 & 98.8 & 97.5 & 98.8 & 98.8 & 73.8 & 0.0 & 0.0 & 87.5 & 98.8 & 98.8 & 98.8 & 98.8 \\
\hline L GOSH_P & 147 & 98.0 & 97.3 & 93.9 & 99.3 & 96.6 & 96.6 & 81.6 & 0.0 & 0.0 & 66.7 & 96.6 & 94.6 & 96.6 & 96.6 \\
\hline Leeds_P & 69 & 89.9 & 95.7 & 89.9 & 95.7 & 98.6 & 98.6 & 87.0 & 98.6 & 98.6 & 53.6 & 98.6 & 91.3 & 98.6 & 97.1 \\
\hline Livpl_P & 46 & 95.7 & 95.7 & 95.7 & 95.7 & 100.0 & 100.0 & 97.8 & 0.0 & 0.0 & 80.4 & 100.0 & 93.5 & 100.0 & 100.0 \\
\hline Manch_P & 63 & 0.0 & 0.0 & 0.0 & 98.4 & 98.4 & 98.4 & 68.3 & 0.0 & 0.0 & 71.4 & 98.4 & 96.8 & 98.4 & 98.4 \\
\hline Newc_P & 27 & 96.3 & 96.3 & 96.3 & 96.3 & 100.0 & 100.0 & 77.8 & 0.0 & 0.0 & 66.7 & 100.0 & 85.2 & 100.0 & 100.0 \\
\hline Nottm_P & 77 & 93.5 & 100.0 & 93.5 & 89.6 & 100.0 & 100.0 & 87.0 & 98.7 & 98.7 & 87.0 & 100.0 & 83.1 & 98.7 & 98.7 \\
\hline Soton_P & 26 & 92.3 & 96.2 & 92.3 & 88.5 & 92.3 & 96.2 & 92.3 & 96.2 & 96.2 & 76.9 & 92.3 & 92.3 & 92.3 & 92.3 \\
\hline UK & 755 & 86.0 & 87.6 & 84.9 & 94.8 & 96.7 & 97.4 & 76.6 & 36.3 & 36.3 & 70.7 & 97.0 & 91.1 & 97.1 & 97.0 \\
\hline
\end{tabular}

BMI - body mass index; SBP - systolic blood pressure; $\mathrm{Hb}$ - haemoglobin; Creat - creatinine; Ferr - ferritin; ESA - erythropoietin stimulating agent; IV - intravenous; Chol - cholesterol; Bicarb - bicarbonate; PTH - parathyroid hormone; Ca - calcium; Phos - phosphate

Table 11.3. Percentage data completeness for dialysis patients $<18$ years old by centre for each variable and total number of patients per centre on 31/12/2016, (annual data return)

\begin{tabular}{|c|c|c|c|c|c|c|c|c|c|c|c|c|c|c|}
\hline \multirow[b]{2}{*}{ Centre } & \multicolumn{14}{|c|}{ Dialysis patients } \\
\hline & $N$ & Height & Weight & BMI & SBP & $\mathrm{Hb}$ & Ferr & ESA & IV iron & Chol & Bicarb & PTH & $\mathrm{Ca}$ & Phos \\
\hline Blfst_P & 8 & 100.0 & 100.0 & 100.0 & 100.0 & 87.5 & 87.5 & 87.5 & 87.5 & 87.5 & 87.5 & 87.5 & 87.5 & 87.5 \\
\hline Brstl_P & 15 & 100.0 & 100.0 & 100.0 & 100.0 & 100.0 & 100.0 & 100.0 & 93.3 & 73.3 & 100.0 & 100.0 & 100.0 & 100.0 \\
\hline Cardf_P & 9 & 88.9 & 100.0 & 88.9 & 88.9 & 100.0 & 100.0 & 0.0 & 0.0 & 66.7 & 100.0 & 100.0 & 100.0 & 100.0 \\
\hline L GOSH_P & 37 & 83.8 & 89.2 & 81.1 & 91.9 & 100.0 & 78.4 & 0.0 & 0.0 & 48.7 & 100.0 & 100.0 & 100.0 & 100.0 \\
\hline Leeds_P & 12 & 75.0 & 100.0 & 75.0 & 100.0 & 100.0 & 91.7 & 100.0 & 100.0 & 83.3 & 100.0 & 100.0 & 91.7 & 100.0 \\
\hline Livpl_P & 9 & 88.9 & 88.9 & 88.9 & 88.9 & 88.9 & 88.9 & 0.0 & 0.0 & 33.3 & 88.9 & 88.9 & 88.9 & 88.9 \\
\hline Manch_P & 25 & 0.0 & 0.0 & 0.0 & 100.0 & 100.0 & 100.0 & 0.0 & 0.0 & 64.0 & 100.0 & 100.0 & 100.0 & 100.0 \\
\hline Newc_P & 5 & 100.0 & 100.0 & 100.0 & 100.0 & 100.0 & 100.0 & 0.0 & 0.0 & 40.0 & 100.0 & 80.0 & 100.0 & 100.0 \\
\hline Nottm_P & 17 & 52.9 & 82.4 & 52.9 & 58.8 & 100.0 & 94.1 & 100.0 & 100.0 & 64.7 & 100.0 & 94.1 & 100.0 & 100.0 \\
\hline
\end{tabular}

BMI - body mass index; SBP - systolic blood pressure; Hb - haemoglobin; Ferr - ferritin; ESA - erythropoietin stimulating agent; IV - intravenous; Chol - cholesterol; Bicarb - bicarbonate; PTH - parathyroid hormone; Ca - calcium; Phos - phosphate

\section{Quarterly data}

All thirteen centres supplied quarterly 2016 data to the UKRR. Completeness of these data is shown for transplant patients in table 11.4 and dialysis patients in table 11.5. PTH and ferritin were not used widely in transplant patients and therefore have been omitted from the table.

\section{Growth}

Height

Figures 11.1 and 11.2 demonstrate that children receiving RRT were shorter for their age and gender than the general childhood population; this was particularly pronounced for children on dialysis. The transplant median height $\mathrm{z}$-score (shown by the dotted line) 
Table 11.4. Percentage data completeness for transplant patients $<18$ years old by centre reporting quarterly laboratory data and total number of patients per centre on 31/12/2016

\begin{tabular}{|c|c|c|c|c|c|c|}
\hline Centre & \multicolumn{6}{|c|}{ Transplant patients } \\
\hline Bham_P & 89 & 90.2 & 55.2 & 90.2 & 90.2 & 89.6 \\
\hline Brstl_P & 40 & 94.0 & 90.6 & 87.9 & 87.9 & 85.9 \\
\hline Cardf_P & 26 & 94.8 & 93.8 & 93.8 & 92.7 & 90.6 \\
\hline Glasg_P & 43 & 82.8 & 82.8 & 82.8 & 82.8 & 82.8 \\
\hline Leeds_P & 69 & 95.7 & 95.7 & 93.3 & 93.3 & 95.3 \\
\hline Livpl_P & 46 & 100.0 & 98.9 & 100.0 & 100.0 & 100.0 \\
\hline Manch_P & 63 & 98.7 & 99.2 & 98.7 & 98.3 & 98.7 \\
\hline Newc_P & 27 & 95.5 & 92.1 & 95.5 & 95.5 & 94.4 \\
\hline Nottm_P & 77 & 90.2 & 86.7 & 86.4 & 86.0 & 86.4 \\
\hline Soton_P & 26 & 94.6 & 76.3 & 80.6 & 78.5 & 80.6 \\
\hline
\end{tabular}

$\mathrm{Hb}$ - haemoglobin

Table 11.5. Percentage data completeness for dialysis patients $<18$ years old by centre reporting quarterly laboratory data and total number of patients per centre on 31/12/2016

\begin{tabular}{|c|c|c|c|c|c|c|c|}
\hline Centre & \multicolumn{7}{|c|}{ Dialysis patients } \\
\hline Bham_P & 23 & 69.8 & 75.0 & 92.7 & 92.7 & 88.5 & 92.7 \\
\hline Brstl_P & 15 & 89.2 & 78.5 & 89.2 & 89.2 & 87.7 & 89.2 \\
\hline Cardf_P & 9 & 100.0 & 97.3 & 100.0 & 100.0 & 97.3 & 100.0 \\
\hline Glasg_P & 17 & 95.1 & 88.5 & 91.8 & 91.8 & 91.8 & 95.1 \\
\hline Leeds_P & 12 & 100.0 & 91.8 & 98.0 & 100.0 & 98.0 & 100.0 \\
\hline Livpl_P & 9 & 100.0 & 100.0 & 100.0 & 100.0 & 23.7 & 100.0 \\
\hline Manch_P & 25 & 100.0 & 100.0 & 100.0 & 100.0 & 100.0 & 100.0 \\
\hline Newc_p & 5 & 100.0 & 89.7 & 100.0 & 100.0 & 96.6 & 100.0 \\
\hline Nottm_P & 17 & 98.4 & 93.5 & 98.4 & 98.4 & 93.5 & 98.4 \\
\hline Soton_P & 4 & 95.7 & 95.7 & 100.0 & 100.0 & 100.0 & 100.0 \\
\hline
\end{tabular}

$\mathrm{Hb}$ - haemoglobin; PTH - parathyroid hormone

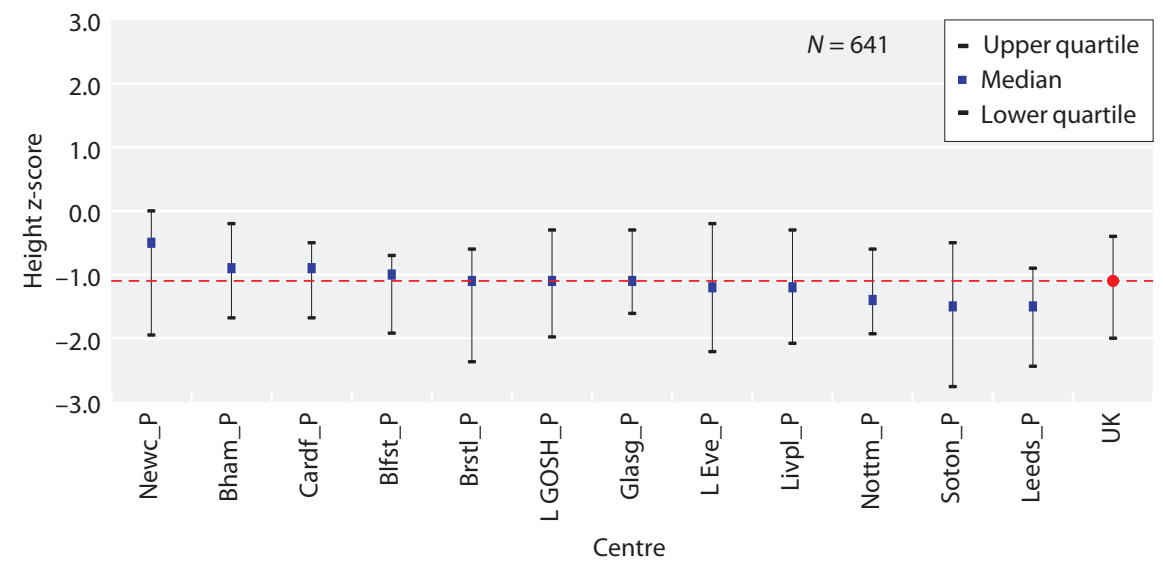

Fig. 11.1. Median height $\mathrm{z}$-scores for transplant patients $<18$ years old on $31 / 12 / 2016$, centre specific and national averages 

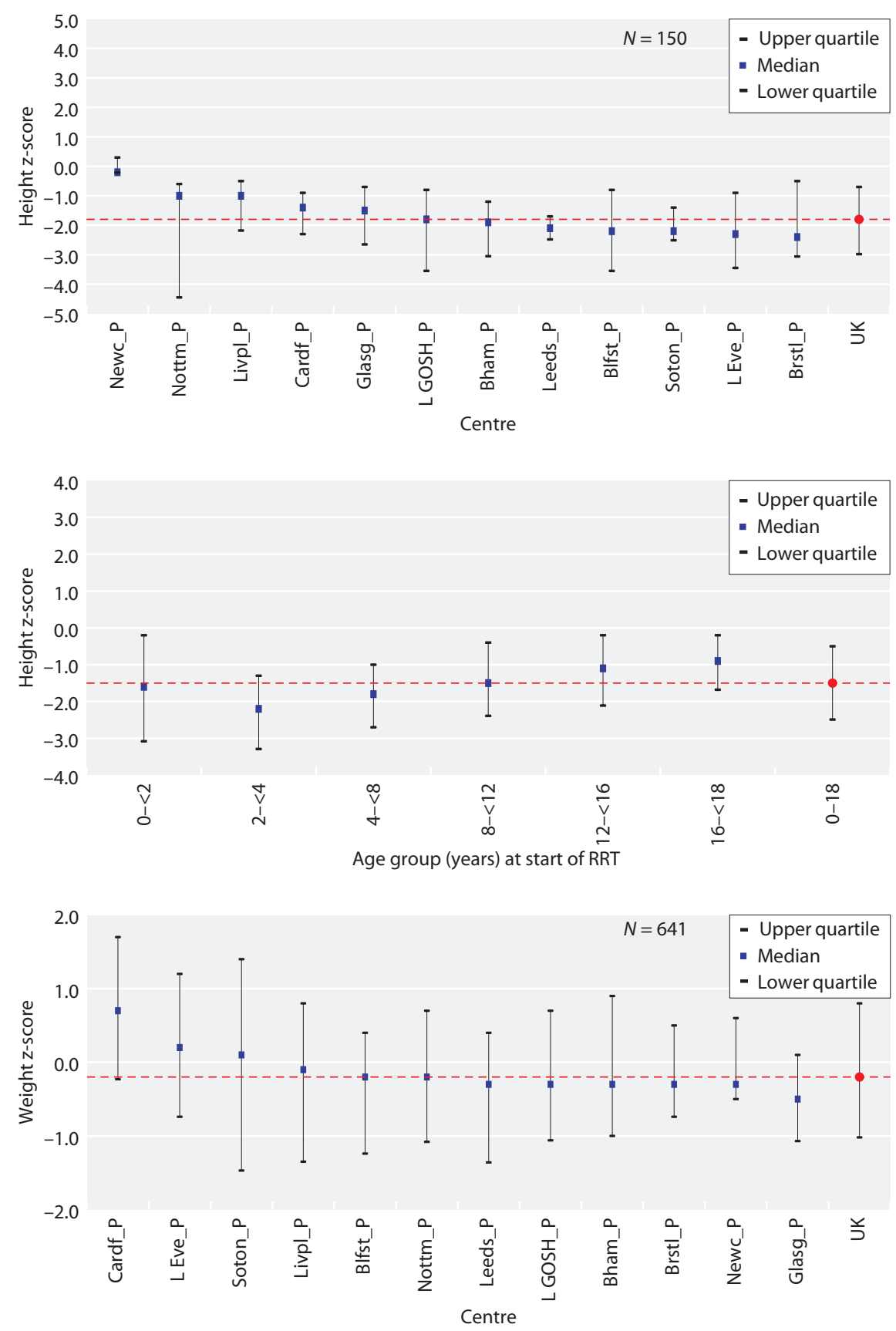

Fig. 11.2. Median height $\mathrm{z}$-scores for dialysis patients $<18$ years old on $31 / 12 / 2016$, centre specific and national averages

Fig. 11.3. Median height $\mathrm{z}$-scores at start of RRT for patients $<18$ years old between 2002 and 2016, by age at start
Fig. 11.4. Median weight $\mathrm{z}$-scores for transplant patients $<18$ years old on $31 / 12 / 2016$, centre specific and national averages was -1.1 and the dialysis median $\mathrm{z}$-score was -1.8 $(p<0.0001)$. Manchester was excluded from both analyses as no 2016 data were submitted to the UKRR for analysis. Examining height $\mathrm{z}$-score at start of RRT (figure 11.3) suggests that children of all age groups were short for their age and height, with an overall median height $\mathrm{z}$-score of -1.5 . As in previous years, younger children, particularly two to less than four year olds, appeared worse off compared with older agegroups.

\section{Weight}

Figure 11.4 shows that paediatric patients with a functioning transplant had a relatively normal weight $\mathrm{z}$-score for age and gender despite a shorter height: overall median z-score was -0.2 . Dialysis patients however had a lower weight for age and gender with a median $\mathrm{z}$ score of -1.2 (figure 11.5), although small numbers hamper further inter-centre analysis. This difference in weight was significant when compared between modality groups $(p<0.0001)$. 

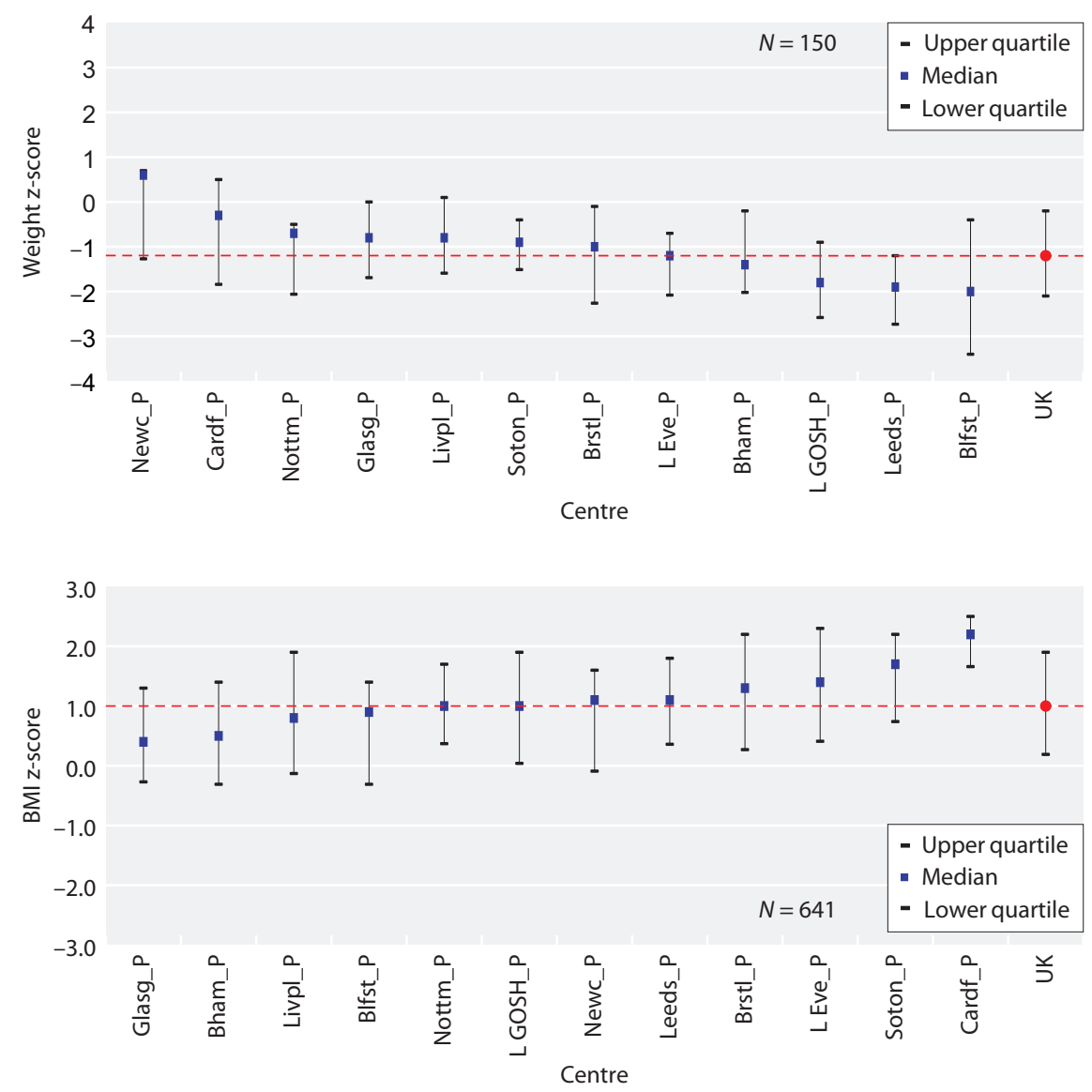

Fig. 11.5. Median weight $\mathrm{z}$-scores for dialysis patients $<18$ years old on $31 / 12 / 2016$, centre specific and national averages

Fig. 11.6. Median BMI z-scores for transplant patients $<18$ years old on $31 / 12 / 2016$, centre specific and national averages

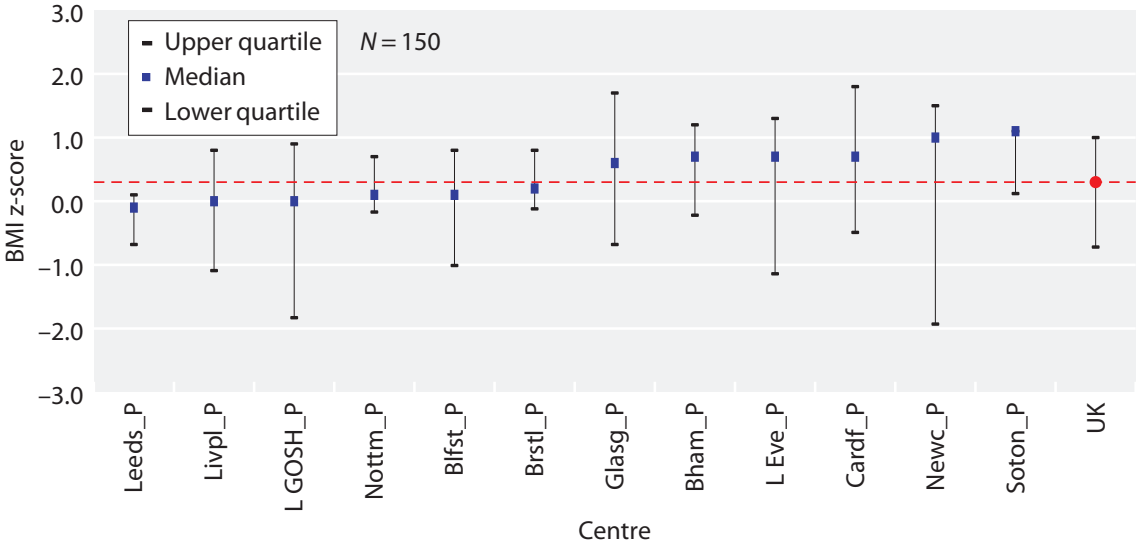

Fig. 11.7. Median BMI z-scores for dialysis patients $<18$ years old on $31 / 12 / 2016$, centre specific and national averages

\section{Cardiovascular risk factor evaluation \\ Obesity}

Examination of BMI by height-age and gender revealed that children in 2016 with functioning transplants had a significantly higher BMI than those receiving dialysis. The median $\mathrm{z}$-score for the respective groups was 1.0 in the transplant group (figure 11.6) and 0.3 in the dialysis group (figure 11.7; $p<0.0001$ ): both median scores fall within the 'normal' BMI category. Manchester was excluded from this analysis given the lack of available height data.

Analysing BMI category by modality, over half of children with transplants (56.6\%) and over three-quarters of dialysis patients $(76.7 \%)$ were classified as having a normal BMI (figure 11.8). Small numbers of patients within both groups were deemed underweight $(<5 \%)$. A much larger proportion of children with a functioning transplant were found to be overweight or obese, 


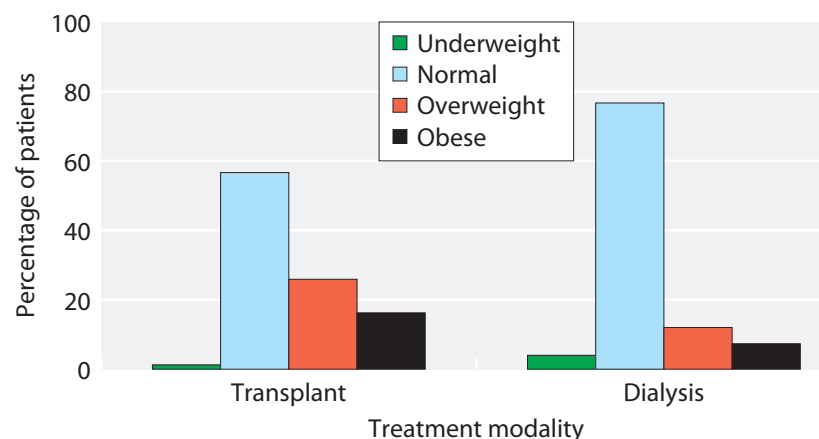

Fig. 11.8. BMI categorisation in children $<18$ years old by modality on $31 / 12 / 2016$

compared with those on dialysis ( $42.2 \%$ vs $19.3 \%$ respectively, $p<0.0001$ ).

Increasing age was associated with higher rates of overweight and obese BMI when analysed using the Mantel-Haenszel method ( $p=0.003$ data not shown). There were no significant differences seen by sex, ethnicity or transplant donor type when comparing proportions of underweight/normal BMI with overweight or obese categories.
Hypertension

Figures 11.9 and 11.10 display median systolic blood pressure (SBP) z-scores by centre for paediatric transplant and dialysis patients respectively, compared with the healthy population. Manchester was excluded from both analyses due to a lack of the 2016 height data needed for standardisation. London Evelina was excluded from figure 11.10 as less than $50 \%$ of patients had available data for both SBP and height variables. In 2016, transplant patients had a relatively normal SBP z-score of 0.3 ; interquartile ranges for only two centres crossed the 90th centile, while over half spanned zero. Compared with transplant patients, dialysis patients had a significantly higher median SBP $z$-score of $0.9(p<0.0001)$, with wide inter-centre variability seen due to the small numbers.

When auditing achievement of SBP standard by modality, a target blood pressure of less than the 90th percentile was achieved in $84.3 \%, 65.2 \%$ and $55.6 \%$ of patients with a functioning transplant, on peritoneal dialysis and on haemodialysis respectively. Small numbers precluded further analysis by strata. This
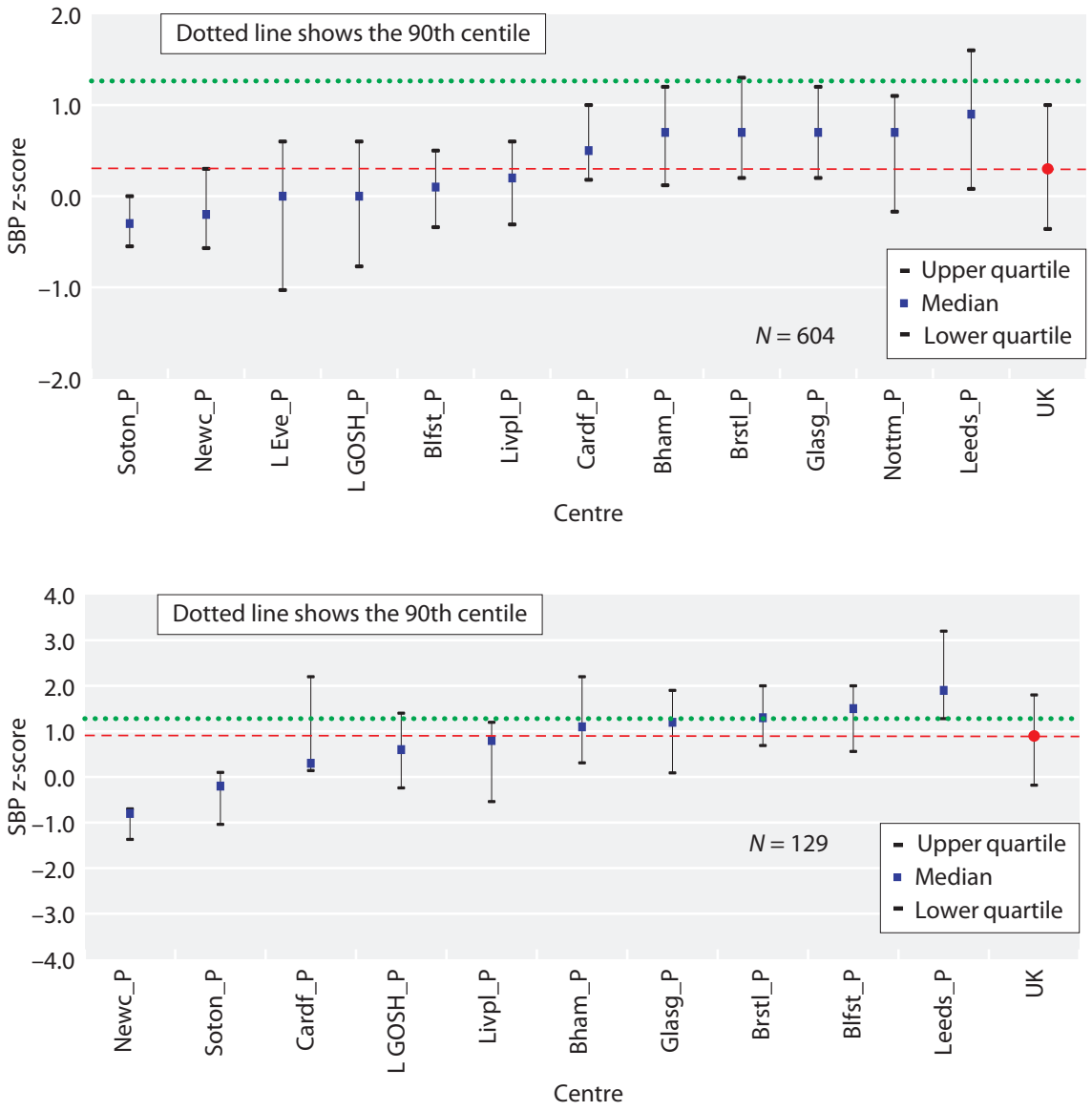

Fig. 11.9. Median SBP z-scores for transplant patients $<18$ years old on $31 / 12 / 2016$, centre specific and national averages

Fig. 11.10. Median SBP $z$-scores for dialysis patients $<18$ years old on 31/12/2016, centre specific and national averages

Plumb/Casula/Magadi/Braddon/Lewis/ Marks/Shenoy/Sinha/Maxwell 
Table 11.6. Percentage of patients $<18$ years old achieving the standard for SBP on 31/12/2016

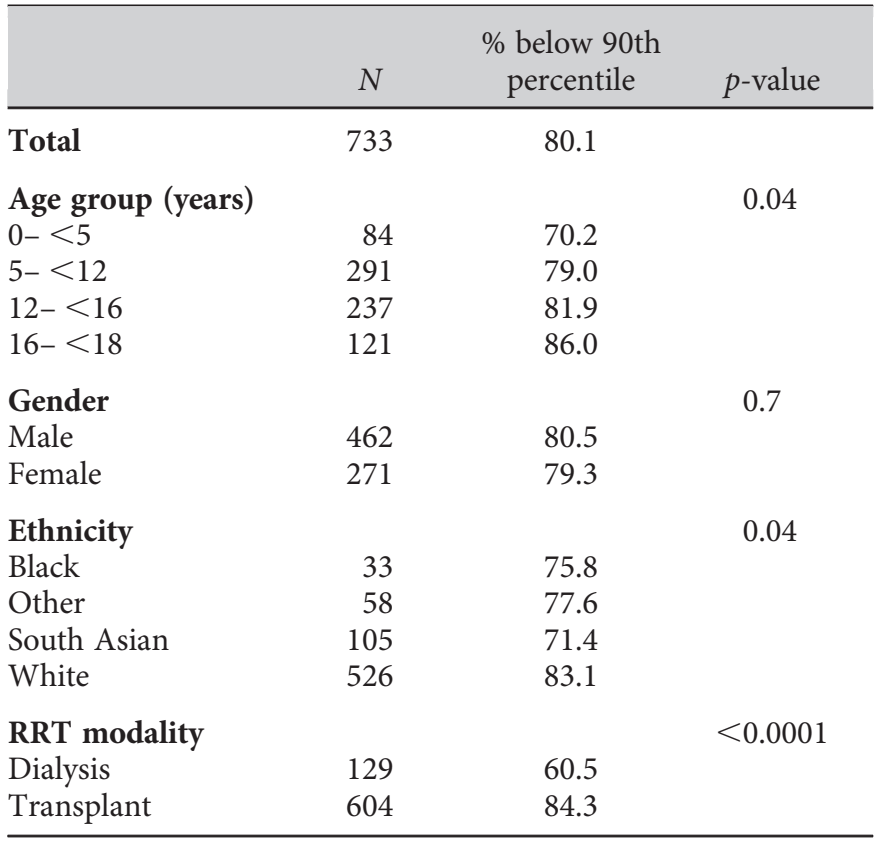

difference was significant between transplant and dialysis patients (table 11.6, $p=<0.0001$ ). Analysing SBP within the total prevalent RRT cohort, significant differences were seen by age and ethnicity. Older children were more likely to achieve the SBP standard compared with younger children, this difference persisted $(p=0.002)$ when analysing only children with a functioning transplant; small dialysis numbers prohibited a comparable analysis. Differences between ethnic groups were also seen. White patients were more likely to achieve the SBP standard (83.1\%), whilst South Asian patients were least likely (71.4\%). No differences were seen when SBP was analysed by dialysis modality or transplant donor type.

Prevalence of cardiovascular risk factors

Table 11.7 demonstrates the proportion of prevalent RRT patients with identified CVRFs: this analysis was restricted to the 553 of 964 (57.4\%) patients with data for all three risk factors. Of those with complete data, over a quarter of patients (27.7\%) had no recorded CVRF, $41.4 \%$ had one CVRF, with under a third of patients (30.9\%) having two or more CVRFs. The most frequently occurring CVRF was high BMI (overweight/ obese categories), affecting $40.5 \%$ of prevalent patients. There were no statistically significant differences in the number of CVRFs according to age-group, ethnicity or modality.

\section{Laboratory and clinical indices - quarterly data}

Tables 11.8 and 11.9 display the median values and interquartile ranges (IQRs) of quarterly laboratory data for prevalent 2016 paediatric transplant and dialysis patients, by centre. For transplant patients, PTH and ferritin data completeness was poor (less than 50\% complete for six and 12 paediatric centres respectively), and was therefore omitted from table 11.8. Overall, transplanted paediatric patients continued to demonstrate good renal allograft function with associated satisfactory biochemistry and anaemia control as a result. For dialysis patients, overall median values for haematological parameters and bicarbonate were satisfactory. While overall median values for corrected calcium and phosphate were normal, there was evidence of hyperparathyroidism (median PTH $16.5 \mathrm{pmol} / \mathrm{L}$ ). Wide variation was noted in median values for ferritin, phosphate and PTH by centre.

Table 11.7. Frequency of number of CVRFs in prevalent RRT patients $<18$ years on $31 / 12 / 2016$

\begin{tabular}{|c|c|c|c|c|c|c|}
\hline Number of CV risk factors & Hypertensive & OW/Obese & Hypercholesterolaemic & $N$ & $\%$ & Total \% \\
\hline \multirow[t]{2}{*}{1} & Yes & No & No & 60 & 10.8 & \\
\hline & No & No & Yes & 70 & 12.7 & \\
\hline \multirow[t]{2}{*}{2} & Yes & Yes & No & 39 & 7.1 & \\
\hline & Yes & No & Yes & 46 & 8.3 & 24.8 \\
\hline $\bar{N}$ & 179 & 224 & 202 & 553 & & \\
\hline Total \% & 32.4 & 40.5 & 36.5 & & & \\
\hline
\end{tabular}

$\mathrm{CV}$ - cardiovascular; OW - overweight 
Table 11.8. Median quarterly laboratory data by centre in prevalent transplant patients $<18$ years old on $31 / 12 / 2016$

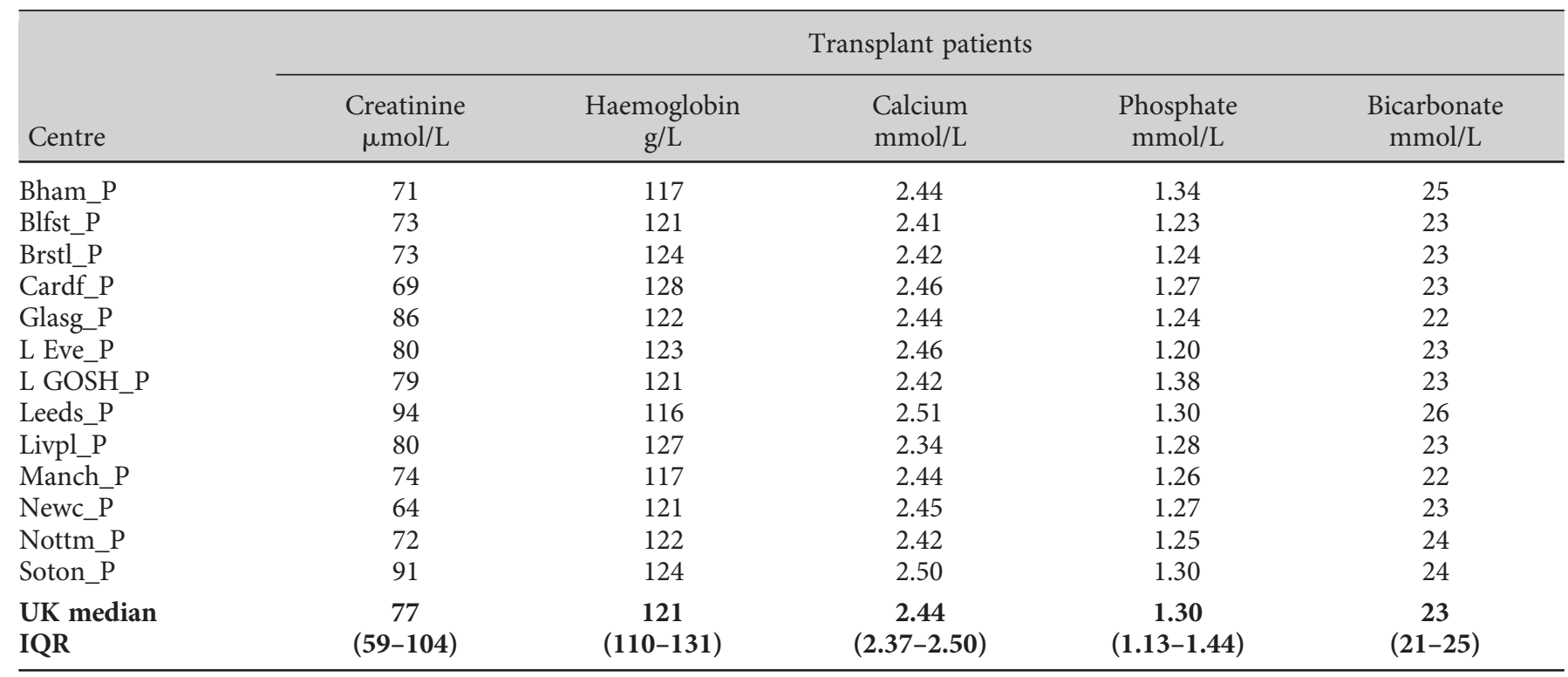

IQR - interquartile range

Table 11.9. Median quarterly laboratory data by centre in prevalent dialysis patients $<18$ years old on $31 / 12 / 2016$

\begin{tabular}{|c|c|c|c|c|c|c|}
\hline \multirow[b]{2}{*}{ Centre } & \multicolumn{6}{|c|}{ Dialysis patients } \\
\hline & $\begin{array}{l}\text { Haemoglobin } \\
\mathrm{g} / \mathrm{L}\end{array}$ & $\begin{array}{c}\text { Ferritin } \\
\mu \mathrm{g} / \mathrm{L}\end{array}$ & $\begin{array}{l}\text { Calcium } \\
\mathrm{mmol} / \mathrm{L}\end{array}$ & $\begin{array}{c}\text { Phosphate } \\
\mathrm{mmol} / \mathrm{L}\end{array}$ & $\begin{array}{c}\text { PTH } \\
\mathrm{pmol} / \mathrm{L}\end{array}$ & $\begin{array}{c}\text { Bicarbonate } \\
\mathrm{mmol} / \mathrm{L}\end{array}$ \\
\hline Bham_P & 111 & 281 & 2.55 & 1.62 & 10.2 & 27 \\
\hline Blfst_P & 122 & 717 & 2.48 & 1.52 & 12.9 & 25 \\
\hline Brstl_P & 111 & 360 & 2.57 & 1.30 & 10.4 & 25 \\
\hline L Eve_P & 105 & 298 & 2.51 & 1.40 & 32.6 & 23 \\
\hline L GOSH_P & 113 & 229 & 2.46 & 1.60 & 9.1 & 25 \\
\hline Leeds_P & 105 & 332 & 2.53 & 2.10 & 36.9 & 24 \\
\hline Livpl_P & 107 & 245 & 2.55 & 1.50 & 13.7 & 25 \\
\hline Manch_P & 107 & 192 & 2.62 & 1.64 & 19.1 & 26 \\
\hline Newc_P & 110 & 328 & 2.57 & 1.39 & 6.9 & 25 \\
\hline
\end{tabular}

PTH - parathyroid hormone; IQR - interquartile range

Table 11.10 demonstrates median eGFR rates for prevalent paediatric transplant recipients by age group and time since transplant. Analysis was performed on 635 of the $755(84.1 \%)$ prevalent transplant patients with height and creatinine data available for 2016. Overall, satisfactory graft function is noted. Younger children tended to have better graft function at any time point post-transplantation compared with older children. By one year, the youngest age group demonstrated an improvement in function, with loss of baseline eGFR ranging between 1.6-11.3\% in older age groups. Median eGFR at one year equates to CKD stage $3 \mathrm{~A}$ for the oldest children, and CKD stage 2 for younger age groups. At five years post-transplantation, loss of baseline eGFR ranges from 5-22.5\% (no data available for patients aged $0-5$ years). Small numbers however have prevented in-depth statistical analysis of these trends. 
Table 11.10. Median estimated glomerular filtration rate (eGFR) by age group and time since transplantation in prevalent transplant patients $<18$ years old on $31 / 12 / 2016$

\begin{tabular}{|c|c|c|c|c|c|c|c|c|}
\hline \multirow{2}{*}{$\begin{array}{l}\text { Time since } \\
\text { transplantation }\end{array}$} & \multicolumn{8}{|c|}{ Age (years) } \\
\hline & \multicolumn{2}{|c|}{$0-<5$} & \multicolumn{2}{|c|}{$5-<12$} & \multicolumn{2}{|c|}{$12-<16$} & \multicolumn{2}{|c|}{$16-<18$} \\
\hline 3 months & 13 & 66 & 32 & 80 & 14 & 64 & 11 & 60 \\
\hline 1 year & 11 & 83 & 35 & 71 & 24 & 63 & 16 & 57 \\
\hline$\geqslant 7$ years & 0 & & 39 & 60 & 87 & 57 & 42 & 53 \\
\hline $\begin{array}{l}\text { Overall median } \\
\text { IQR }\end{array}$ & 33 & $\begin{array}{c}77 \\
(51-99)\end{array}$ & 267 & $\begin{array}{c}66 \\
(53-84)\end{array}$ & 219 & $\begin{array}{c}59 \\
(45-74)\end{array}$ & 116 & $\begin{array}{c}54 \\
(42-70)\end{array}$ \\
\hline
\end{tabular}

IQR - interquartile range

\section{Laboratory and clinical indices - annual data}

Laboratory data described below are for the prevalent RRT patients aged less than 18 years and managed in paediatric centres in 2016. Achievement of standards was calculated using one value per patient, the most recent value for 2016 was used in each case. Interpretation of inter-centre variation is limited when stratified by RRT modality given the small numbers.

\section{Haemoglobin and ferritin}

The proportion of patients with a functioning renal transplant achieving the haemoglobin standard in 2016 was $91.1 \%$, compared with $71 \%$ of haemodialysis and $77 \%$ of peritoneal dialysis patients. The proportions of transplant and dialysis (both modalities) patients achieving the national haemoglobin standard has been consistent over the last ten years: $72-75 \%$ of dialysis patients achieved the standard over this time compared with 92-93\% of transplant patients.

\section{Calcium}

The proportion of haemodialysis patients under 18 years achieving the calcium standard was slightly higher in 2016 at $79.3 \%$ compared to the previous year. Less than $5 \%$ of patients were hypocalcaemic and $16 \%$ were reportedly hypercalcaemic. Of the peritoneal dialysis cohort, a similar proportion of patients (69.1\%) achieved the calcium standard in 2016, compared with the previous year. No PD patients were hypocalcaemic.

\section{Phosphate}

Just over half of prevalent haemodialysis and peritoneal dialysis patients achieved the phosphate standard in 2016: $53.8 \%$ and 58.8\% respectively. Similar proportions of each patient group were hyperphosphataemic (32.1\% of HD; $35.1 \%$ of PD patients). No differences were seen in attainment of standard by age group.

\section{Parathyroid hormone}

Little change was noted in 2016 with regards to the overall attainment of satisfactory PTH values compared with the previous year. The proportion of transplant patients aged $<18$ years maintaining a $\mathrm{PTH}$ within an acceptable range was $80.7 \% ; 19.3 \%$ demonstrated evidence of hyperparathyroidism. For HD patients, more patients had hyperparathyroidism (59.1\%) than acceptable levels (41\%). This trend was similar in PD patients: $64.6 \%$ of the cohort had documented hyperparathyroidism, $35.4 \%$ achieved the standard.

\section{Bicarbonate}

In $2016,86.2 \%$ of prevalent transplant patients, $73.6 \%$ of haemodialysis patients and $68 \%$ of peritoneal dialysis patients had a bicarbonate level within the agreed standard range. Peritoneal dialysis patients had the highest proportion of patients with levels exceeding the standard (30.9\%) compared to $10.4 \%$ of HD and $4.9 \%$ of transplant patients. Fewer patients were acidotic: the proportion was highest in the HD group (16.0\%), followed by transplant recipients $(8.9 \%)$ and $\mathrm{PD}$ patients $(1.0 \%)$.

\section{Discussion}

This chapter offers an insight into the haematological and biochemical management of established renal failure for children aged less than 18 years undergoing RRT in 
paediatric centres. Comparison of laboratory and clinical indices in most cases are made by modality type and centre, although small numbers limit in-depth analysis at this level. Furthermore, discrepancies in attainment of nationally agreed standards relevant to the management of ERF will help to identify areas for future quality improvement work. It is hoped that data from this report can continue to support the paediatric nephrology community to improve clinical care and outcomes for patients.

\section{Quarterly data}

All centres submitted quarterly data to the UKRR. This serves to strengthen future research, with more timepoints available for analyses of growth and allograft function. Median creatinine data for paediatric transplant patients suggests good overall allograft function, with associated normal range values seen for haematological and biochemistry parameters. Dialysis patients demonstrated good control of anaemia, acidosis and calcium, although wide variation in median phosphate and PTH values were seen across centres. The high median PTH value of $16.5 \mathrm{pmol} / \mathrm{L}$ suggests management of hyperparathyroidism remained an ongoing challenge for this cohort. This has improved however from 2015 data (previously $21.0 \mathrm{pmol} / \mathrm{L}$ ).

\section{Highlights from the 2016 data}

Data completeness for 2016 fell, thus hampering the ability to fully report outcomes for all data items collected by the UKRR. Key variables including height, weight and systolic blood pressure were of good completeness. Lack of data was particularly problematic for ESA, IV iron and growth hormone use, as such analyses included in previous years have been removed from this report. Cholesterol was of sufficient completeness to be included in the CVRF analysis. It is difficult to be certain of the reasons for reduced data completeness, but this needs to be explored.

\section{Growth}

Height at start of RRT, as well as for prevalent children on RRT in 2016 remained lower than that of the healthy UK paediatric population. This was strongly associated with RRT modality, with dialysis patients demonstrating lower height $\mathrm{z}$-scores than transplanted children.

As expected, younger children at start of RRT tended to fare worse in terms of height z-score compared with older children, although given the small numbers and associated wide interquartile ranges, this was based on the observed trend. It is interesting to note that even young people reaching ERF at the end of adolescence were shorter than their peers (median $\mathrm{z}$-score -0.9 ), although this observation does not consider duration of kidney disease, primary renal diagnosis or associated extra-renal comorbidity and final height as adults was not known.

In terms of weight, transplant patients had similar z-scores to those seen in the healthy population. Dialysis patients by comparison were underweight, with a median weight $\mathrm{z}$-score of -1.2 . As in previous years, a relatively short height with near-normal weight attainment meant that a high proportion of transplanted children were in the overweight or obese BMI range $(42.1 \%$ vs $19.3 \%$ dialysis). Given that being overweight or obese was the most frequently reported cardiovascular risk factor within the paediatric RRT population, further analysis of this population is warranted to understand how this risk can be modified and reduced.

\section{Cardiovascular risk factor evaluation}

As in previous years, many prevalent RRT patients have one or more modifiable cardiovascular risk factors. For transplanted children, high BMI appears particularly problematic, with $42.1 \%$ of the total cohort reportedly overweight or obese for their height-age. For children receiving dialysis, management of hypertension was a greater issue: only $56 \%$ of haemodialysis and $66 \%$ of peritoneal dialysis patients achieved a systolic blood pressure below the 90th percentile in 2016. Given that cardiac disease remained one of the most common causes of death for young UK adults with ERF [10], timely consideration and treatment of these risk factors, together with maximizing access to transplantation, is important for the long-term health of this population. It would also be of value to further investigate patient and disease characteristics of children who have no CVRF whilst on RRT.

\section{Laboratory and clinical indices}

Attainment of standards for laboratory measures were similar to previous years for both transplant and dialysis patients. The updated 2015 NICE guidance on the management of anaemia in CKD has advised that ferritin alone should not be routinely used to assess iron deficiency status. In view of this, isolated ferritin data has not been interpreted this year in the context of anaemia management, focusing only on assessment of maximum iron levels. In previous years, completeness of ferritin data has been variable by centre and therefore 
difficult to interpret; this is likely to reflect alternative methods of anaemia assessment being used in lieu of ferritin. Recommendations now suggest that assessment of anaemia and responsiveness to treatment should be based on the percentage of hypochromic red blood cells (\%HRC), reticulocyte haemoglobin content, or transferrin saturation in conjunction with ferritin. Serum ferritin can be used to guide maximum iron levels and IV iron therapy. Information from centres on how best to collect haematological data relating to anaemia management for future reports is welcomed.

\section{References}

1 The Renal Association. Treatment of adults and children with renal failure: standards and audit measures. Third edition. 2002. Available from: https://renal.org/wp-content/uploads/2017/06/Renal_Association_ Standards_3rd_Edition_2002-2007.pdf (last accessed 17th May 2018)

2 Cole TJ, Flegal KM, Nicholls D, Jackson AA. Body Mass Index cut offs to define thinness in children and adolescents: international study. BMJ 2007;335(7612):194

3 Freeman JV, Cole TJ, Chinn S et al. Cross-sectional stature and weight reference curves for the UK, 1990. Arch Dis Child 1995;73:1 7-24

4 BAPN Standards for Hypertension in Paediatric Renal Transplant Recipients, 2011. Available from: https://renal.org/wp-content/uploads/ 2017/07/bapn-standards-for-hypertension-in-renal-transplant-recipients. pdf (last accessed 15th May 2018)

5 National High Blood Pressure Education Program Working Group on High Blood Pressure in Children and Adolescents. The Fourth Report on the Diagnosis, Evaluation, and Treatment of High Blood Pressure in Children and Adolescents. Pediatrics 2004;114(2):55 5-76

\section{Future work}

The UKRR has approved the use of data for an analysis of risk factors implicated in the rate of eGFR function decline post-transplantation in paediatric and young adult patients. Additionally, potential quality improvement projects based on findings of previous years' reports have been discussed amongst BAPN members at their 2018 annual meeting. For the UKRR, suggestions on how to improve the process of timely accurate and complete data returns from paediatric centres are welcomed.

Conflicts of interest: the authors declare no conflicts of interest

6 Expert panel on integrated guidelines for cardiovascular health and risk reduction in children and adolescents: summary report. Pediatrics 2011 Dec. 128(suppl 5):S21 3-56. doi: 10.1542/peds.2009-2107C

7 Kidney Disease Improving Global Outcomes (KDIGO). Clinical Practice Guideline for Lipid Management in Chronic Kidney Disease. Kidney International Supplements 2013;3(3):1-305

8 NICE clinical guideline NG8. Chronic Kidney Disease: Managing anaemia. London: National Institute for Health and Clinical Excellence, 2015. https://www.nice.org.uk/guidance/ng8/resources/chronic-kidney-diseasemanaging-anaemia-pdf-51046844101 (last accessed 8th May 2018)

9 Schwartz GJ, Munoz A, Schneider MF, Mak RH, Kaskel F, Warady BA, Furth SL. New equations to estimate GFR in children with CKD. Journal of the American Society of Nephrology 2009;Mar 1;20(3):629-37

10 Methven S, Steenkamp R, Fraser S. UK Renal Registry 19th Annual Report: Chapter 5. Survival and causes of death in UK adult patients on renal replacement therapy in 2015: National and Centre-specific analyses. Nephron. 2017;137(suppl 1):117-50 
